\title{
ALTERAÇÕES EM PROPRIEDADES DE SOLO ADUBADO COM DOSES DE COMPOSTO ORGÂNICO SOB CULTIVO DE BANANEIRA ${ }^{1}$
}

\author{
ERVAL RAFAEL DAMATTO JUNIOR ${ }^{2}$, ROBERTO LYRA VILLAS BÔAS ${ }^{3}$, SARITA LEONEL ${ }^{4}$, \\ DIRCEU MAXIMINO FERNANDES ${ }^{5}$
}

\begin{abstract}
RESUMO - Com o intuito de avaliar os efeitos de diferentes doses de composto orgânico nas propriedades químicas do solo cultivado com bananeira 'Prata-anã' (Musa AAB), foi desenvolvido o presente trabalho na Faculdade de Ciências Agronômicas - UNESP, Botucatu-SP. O plantio foi realizado no mês de novembro de 2002, com mudas convencionais, adotando-se o espaçamento de 2,5 x $2,5 \mathrm{~m}$. O composto orgânico foi produzido com serragem de madeira e esterco de bovino, sendo os tratamentos empregados constituídos das seguintes doses de composto: $\mathrm{T} 1=0 \mathrm{~g}_{\text {planta }}{ }^{-1}$ de $\mathrm{K}_{2} \mathrm{O}$ (dose zero do composto - Testemunha); $\mathrm{T} 2=98,5 \mathrm{~g}_{\text {planta }}{ }^{-1}$ de $_{2} \mathrm{O}$ (43 kg planta ${ }^{-1}$ de composto); $\mathrm{T} 3$

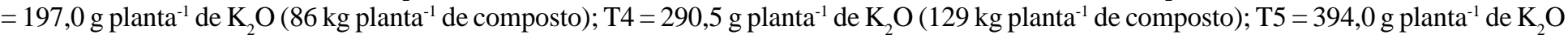
(172 $\mathrm{kg}_{\text {planta }}{ }^{-1}$ de composto), sendo essas doses calculadas de acordo com o teor de potássio presente no mesmo. O delineamento experimental adotado foi em blocos casualizados, com 5 tratamentos, 5 repetições e 2 plantas por parcela. Os dados foram submetidos à análise de variância e à análise de regressão. Aos quatro meses após a aplicação da última parcela da adubação com composto orgânico, realizou-se amostragem de solo da camada de 0 a $20 \mathrm{~cm}$ e foram avaliadas as propriedades químicas do solo. A adubação orgânica promoveu incrementos no pH, matéria orgânica, fósforo, cálcio, soma de bases, CTC e saturação por bases do solo.
\end{abstract}

Termos para indexação: Musa sp., Prata-anã, adubação orgânica, fertilidade do solo, potássio.

\section{CHANGES IN SOIL PROPERTIES MANAGED WITH ORGANIC COMPOST RATES, UNDER BANANA PLANT}

\begin{abstract}
ABTRACT - Aiming to evaluate the effects of different organic compost rates in chemical properties of soil cultivated with banana plants 'Prata-anã' (Musa AAB), this present work was carried out at "Faculdade de Ciências Agronômicas - UNESP", Botucatu-SP. Plants were placed in the prepared area in November 2002, at 2,5 x 2,5 m spacing between plants. The organic compost was produced using wood residue and bovine manure and the treatments were constituted by different compost rates: $\mathrm{T} 1=0 \mathrm{~g} \mathrm{plant}^{-1}$ of $_{2} \mathrm{O}$ (zero of compost); $\mathrm{T} 2=98,5 \mathrm{~g}$

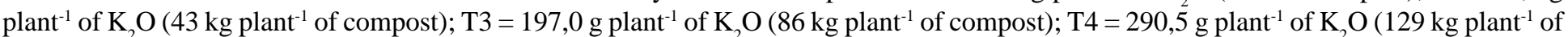
compost); T5 = 394,0 $\mathrm{g}$ plant $^{-1}$ of $\mathrm{K}_{2} \mathrm{O}\left(172 \mathrm{~kg}\right.$ plant $^{-1}$ of compost). Rates were calculated based on the amount of potassium contained in the compost. The experiment was arranged in a randomized block design, with 5 treatments, 5 replications and 2 plants per plot. Data were submitted to variance analysis and to regression analysis. Four months after the last compost application, the soil was sampled at $20 \mathrm{~cm}$ deep and the chemical properties of the soil were evaluated. The organic fertilization increased $\mathrm{pH}$, organic matter, phosphorus, calcium, the sum of bases, CTC, and the base saturation of the soil.
\end{abstract}

Index terms: Musa sp., Prata-anã, organic fertilization, soil fertility, potassium.

A banana é uma das frutas mais consumidas no mundo, explorada na maioria dos países tropicais, sendo atingida uma produção mundial de 70,6 milhões de toneladas em 2006 (Agrianual, 2006). A produção de bananas no sistema orgânico aparece como alternativa para que o produto final possa atingir uma parcela de consumidores específica, com maior poder aquisitivo (Agrianual, 2001).

Hewitt (1955), Twyford \& Walmsley (1974) e Neves et al. (1991) mencionam que a bananeira, independentemente da cultivar, extrai maiores quantidades de potássio que nitrogênio durante o ciclo de formação da planta. Essa extração, do período juvenil até o lançamento do cacho, é bastante acentuada, quando são verificadas elevadas retiradas de K, N, Ca, P e Mg (Martin-Prével, 1964; Gallo et al., 1972), sendo que, em condições brasileiras, Hiroce et al. (1977) determinaram a extração de macronutrientes em gramas por tonelada de frutos frescos de bananeira 'Nanicão' para uma produção média de 68t/ha, observando a seguinte ordem de grandeza: 8899, 2060, 309, 288, 282 e 53g/t, respectivamente para K, N, S, Ca P e Mg.

Dentre os resíduos gerados na agropecuária, o esterco bovino é um dos que contém quantidades variáveis de nutrientes e que pode ser usado na agricultura, na substituição ou complementação da adubação química. A serragem de madeira também é um resíduo que pode ser aproveitado no processo de compostagem, servindo como fonte de carbono, contudo sua decomposição é mais lenta que materiais baseados em celulose, que são decompostos três vezes mais rápido em relação às partes lenhosas ricas em taninos (Larcher, 2000). Essa diferença no tempo de decomposição dos resíduos assegura um fluxo contínuo de nutrientes no solo.

Campo Dall'Orto et al. (1996) salientam que o principal efeito da adubação orgânica é na melhoria das propriedades físicas e químicas do solo. No entanto, conforme os mesmos autores, a liberação dos nutrientes dos adubos orgânicos é mais lenta que a dos adubos minerais solúveis, pois é dependente da mineralização da matéria orgânica. De acordo com a Comissão de Fertilidade do Solo do Estado de Minas Gerais (1989), para alguns materiais orgânicos, a conversão do nitrogênio da forma orgânica para a mineral ocorre $50 \%$ no primeiro ano, $20 \%$ no segundo ano e $30 \%$ após o segundo ano. Bartz et al. (1995) citaram que 50\% do nitrogênio aplicado ao solo mineraliza no primeiro cultivo e $20 \%$ no segundo, enquanto o fósforo, mineraliza $60 \%$ no primeiro cultivo e $20 \%$ no segundo. Já o potássio, por não se encontrar ligado a nenhum composto orgânico, tem sua liberação quase que total logo após sua aplicação.

\footnotetext{
${ }^{1}$ (Trabalho 04-2006). Recebido: 06-01-2006. Aceito para publicação: 25-08-2006.

${ }^{2}$ Eng. Agr. Doutorando em Horticultura da Faculdade de Ciências Agronômicas/UNESP. Depto. de Recursos Naturais/Solos, Cep: 18610-307 - BotucatuSP. E-mail: ervaljr@fca.unesp.br. Bolsista Fapesp.

${ }_{3}^{3}$ Prof. Dr. do Depto. de Recursos Naturais - Solos da Faculdade de Ciências Agronômicas/UNESP, Cep: 18610-307 - Botucatu-SP. E-mail: rlvboas@fca.unesp.br.

${ }^{4}$ Prof $^{a}$. Dra ${ }^{a}$ do Depto. de Produção Vegetal - Horticultura da Faculdade de Ciências Agronômicas/UNESP, Cep: 18610-307 - Botucatu-SP. E-mail: sarinel@fca.unesp.br.

${ }^{5}$ Prof. Dr. do Depto. de Recursos Naturais - Solos da Faculdade de Ciências Agronômicas/UNESP, Cep: 18610-307 - Botucatu-SP. E-mail: dmfernandes@fca.unesp.br.
}

Rev. Bras. Frutic., Jaboticabal - SP, v. 28, n. 3, p. 546-549, Dezembro 2006 
Tem sido recomendada a aplicação de matéria orgânica humificada ao solo como uma maneira de controlar a toxidez causada às culturas agronômicas por certos elementos encontrados em quantidades acima do normal, como o alumínio, ferro e manganês, uma vez que o húmus tem a propriedade de fixar, complexar ou quelatar esses elementos (Kiehl, 1985). Com relação à acidez do solo, Hunter et al. (1995) e Wong et al. (1995) comentam que a matéria orgânica do solo pode apresentar um efeito semelhante ao da calagem, na correção da acidez e na neutralização de níveis tóxicos de alumínio. $\mathrm{O}$ aumento do $\mathrm{pH}$ do solo devido à adição de resíduos orgânicos tem sido atribuído à própria adsorção de hidrogênio e alumínio na superfície do material orgânico (Hoyt \& Turner, 1975). Este trabalho objetivou avaliar os efeitos da aplicação de doses de composto orgânico nas propriedades químicas do solo após um ciclo de cultivo com bananeira 'Prata-anã'.

O experimento foi realizado na Faculdade de Ciências Agronômicas - UNESP, Câmpus de Botucatu-SP, que apresenta as seguintes coordenadas geográficas: Latitude $22^{\circ} 52^{\prime} 55^{\prime \prime} \mathrm{S}$ Longitude $48^{\circ} 26^{\prime} 22^{\prime \prime} \mathrm{W}$ e altitude $786 \mathrm{~m}$, sendo o clima classificado temperado quente (mesotérmico) com chuvas no verão e seca no inverno (Cwa - Köppen), e a temperatura média mais quente, superior a $22{ }^{\circ} \mathrm{C}$ (Cunha et al., 1999). O solo da área foi classificado como terra roxa estruturada - unidade lageado, álico, textura argilosa (Carvalho et al., 1983), atualmente Nitossolo Vermelho, segundo os critérios da Embrapa (1999).

Para a produção do composto, foram construídas pilhas de compostagem utilizando materiais com alto teor de carbono (serragem de madeira, com teores de $\mathrm{C}=551,6 \mathrm{~g} \mathrm{~kg}^{-1} \mathrm{e} \mathrm{N}=5,4 \mathrm{~g} \mathrm{~kg}$ $\left.{ }^{1}\right)$ e outro com alto teor de nitrogênio (esterco bovino, com teores de $\mathrm{C}=295,8 \mathrm{~g} \mathrm{~kg}^{-1} \mathrm{e} \mathrm{N}=23,5 \mathrm{~g} \mathrm{~kg}^{-1}$ ), que foram misturados em betoneira, visando a atingir a relação carbono/nitrogênio de 30/1.

A área experimental foi preparada dois meses antes do plantio, onde se realizou: aração, gradagem, seguidos de calagem em área total, conforme a análise de solo, objetivando elevar a saturação por bases a $60 \%$ e o teor de magnésio acima de $9 \mathrm{mmol} / \mathrm{dm}^{3}$ (Raij et al., 1997), utilizando-se de calcário dolomítico (PRNT =91). Em seguida, foi aplicado em área total chorume de esterco bovino, proveniente dos mesmos animais que forneceram o esterco para a produção do composto orgânico, equivalente a $138 \mathrm{mil} \mathrm{L} \mathrm{ha}^{-1}$, o qual apresentava a seguinte composição (em g $\left.100 \mathrm{~mL}^{-1}\right): 0,05 \mathrm{de} \mathrm{N} ; 0,02$ de $_{2} \mathrm{O}_{5} ; 0,04$ de $\mathrm{K}_{2} \mathrm{O} ; 0,29$ de $\mathrm{MO}$; 0,03 de $\mathrm{Ca}$; e 0,01 de $\mathrm{Mg}$.

As covas para o plantio tiveram as dimensões de $60 \mathrm{~cm} \mathrm{de}$ diâmetro por $80 \mathrm{~cm}$ de profundidade, as quais foram preparadas com $7 \mathrm{~L}$ de esterco de curral curtido e $300 \mathrm{~g}$ de termofosfato. O plantio foi realizado no mês de novembro de 2002, com mudas retiradas de bananeiras da cv. Prata-anã, adotando-se o espaçamento de $2,5 \mathrm{~m}$ entre linhas e 2,5 m entre plantas. Foi instalado um sistema de irrigação localizada (microaspersão), com vazão de emissores de 40 $\mathrm{L} \mathrm{h}^{-1}$, sendo a lâmina de água calculada de acordo com a evaporação do tanque Classe A. O delineamento experimental adotado foi em blocos casualizados, com 5 tratamentos, 5 repetições e 2 plantas por parcela.

Os tratamentos empregados foram constituídos de diferentes doses de composto orgânico: $\mathrm{T} 1=0 \mathrm{~g}_{\text {planta }}{ }^{-1} \mathrm{de}_{2} \mathrm{O}$ (dose zero do composto - Testemunha); $\mathrm{T} 2=98,5 \mathrm{~g}$ planta $^{-1} \mathrm{de} \mathrm{K}_{2} \mathrm{O}$ (43 kg planta ${ }^{1}$ de composto); T3 $=197,0 \mathrm{~g}_{\text {planta }}{ }^{-1} \mathrm{de}_{\mathrm{K}} \mathrm{O}\left(86 \mathrm{~kg}\right.$ planta $^{-1} \mathrm{de}$ composto); $\mathrm{T} 4=290,5 \mathrm{~g}$ planta $^{-1}$ de $\mathrm{K}_{2} \mathrm{O}\left(129 \mathrm{~kg}\right.$ planta ${ }^{-1} \mathrm{de}$ composto $) ; \mathrm{T} 5=394,0 \mathrm{~g}_{\text {planta }}{ }^{-1}$ de $\mathrm{K}_{2} \mathrm{O}\left(172 \mathrm{~kg}\right.$ planta $^{-1} \mathrm{de}$ composto), sendo essas doses calculadas de acordo com o teor de potássio presente no mesmo e que, por sua vez, se baseou no Boletim 100 (Raij et al., 1997). A adubação anual, utilizando o composto como fonte de nutrientes, iniciou-se três meses após o plantio, sendo dividida em 5 parcelas, aplicadas ao redor das plantas a cada dois meses, nas quantidades determinadas para cada tratamento. $\mathrm{O}$ composto empregado como fonte de nutrientes apresentava as seguintes características: $\mathrm{pH}=7,5$; relação $\mathrm{C} / \mathrm{N}=25 / 1$; Umidade $=$
$62 \% ; \mathrm{MO}=77 \%$. Apresentou dados em $\mathrm{g} \mathrm{kg}^{-1}(\mathrm{x} 10): \mathrm{N}=1,75 ; \mathrm{P}_{2} \mathrm{O}_{5}=$ 1,$44 ; \mathrm{K}_{2} \mathrm{O}=0,60 ; \mathrm{Ca}=1,96 ; \mathrm{Mg}=0,33 ; \mathrm{S}=0,30 ; \mathrm{C}=42,78 ;$ e em mg $\mathrm{kg}^{-1}: \mathrm{Fe}=2450 ; \mathrm{Cu}=56 ; \mathrm{Mn}=114 ; \mathrm{Na}=1600 ; \mathrm{Zn}=112$.

As características químicas iniciais do solo, na camada de 0 a $20 \mathrm{~cm}$, foram as seguintes: $\mathrm{pH}\left(\mathrm{CaCl}_{2}\right)=5,9 ; \mathrm{MO}=28 \mathrm{~g} \mathrm{dm}^{-3} ; \mathrm{P}$ (resina) $=102 \mathrm{mg} \mathrm{dm}^{-3} ; \mathrm{H}+\mathrm{Al}=31 \mathrm{mmol} \mathrm{dm}^{-3} ; \mathrm{K}=4,2 \mathrm{mmol} \mathrm{dm}^{-3} ; \mathrm{Ca}$ $=65 \mathrm{mmol}_{\mathrm{c}} \mathrm{dm}^{-3} ; \mathrm{Mg}=22 \mathrm{mmol} \mathrm{dm}_{\mathrm{c}}^{-3} ; \mathrm{SB}=91 \mathrm{mmol}_{\mathrm{c}} \mathrm{dm}^{-3} ; \mathrm{CTC}=122$ $\mathrm{mmol}_{\mathrm{c}} \mathrm{dm}^{-3} ; \mathrm{V}=75 \% ; \mathrm{B}=0,19 \mathrm{mg} \mathrm{dm}^{-3} ; \mathrm{Cu}=3,8 \mathrm{mg} \mathrm{dm}^{-3} ; \mathrm{Fe}=23 \mathrm{mg}$ $\mathrm{dm}^{-3} ; \mathrm{Mn}=11,6 \mathrm{mg} \mathrm{dm}^{-3} ; \mathrm{Zn}=2,3 \mathrm{mg} \mathrm{dm}^{-3}$

Os efeitos da adubação orgânica no solo foram avaliados por meio de análises químicas, sendo que, em cada parcela experimental, retiraram-se 5 subamostras de solo $(0-20 \mathrm{~cm})$ na área de aplicação do composto, para formar uma amostra composta por parcela. As amostras coletadas no término da primeira colheita (março de 2004), ou seja, quatro meses após a aplicação da última parcela da adubação anual foram encaminhadas para o Laboratório de Fertilidade do Solo do Departamento de Recursos Naturais - Solo para a determinação dos teores de macronutrientes disponíveis (potássio, fósforo, cálcio e magnésio), micronutrientes (boro, cobre, ferro, manganês e zinco), além do pH, teor de matéria orgânica (M.O.), soma de bases (SB), capacidade de troca catiônica (CTC) e saturação por bases (V). As amostras foram secas em estufa e analisadas conforme metodologia preconizada por Raij \& Quaggio (1983). Os dados foram submetidos à análise de variância e à análise de regressão.

A aplicação de composto orgânico proporcionou efeito significativo nas propriedades químicas do solo, exceto para potássio e magnésio (Tabela 1). Assim, observou-se aumento de forma linear em relação ao $\mathrm{pH}$ do solo, atingindo valores máximos nos tratamentos com as maiores doses de composto adicionado ao solo. Isso evidencia que o aumento de matéria orgânica no solo por meio da adição de composto tendeu a elevar o pH do solo, conforme Hunter et al. (1995), Wong et al. (1995) e Hoyt \& Turner (1975), uma vez que a matéria orgânica no solo indisponibiliza o alumínio, fazendo com que o pH do solo se eleve. Essa elevação no pH do solo é importante na diminuição da incidência de "mal-do-Panamá" (Cordeiro, 1999), doença fúngica, causado por Fusarium oxysporum que diminui a produção e a vida útil do bananal.

Os teores de matéria orgânica no solo aumentaram linearmente (Tabela 1) com as doses de composto aplicado. Na fase inicial do desenvolvimento das bananeiras, a matéria orgânica estimula o desenvolvimento das raízes, além de fornecer o nitrogênio, que nesta fase é de fundamental importância ao crescimento da planta (Moreira, 1987).

Os teores de fósforo apresentaram elevação com o aumento das doses de composto, sendo encontrados os menores teores na testemunha ( $54 \mathrm{mg} \mathrm{dm}^{-3}$ ) e os maiores teores nos tratamentos com as doses de 129 e $172 \mathrm{~kg}$ planta $^{-1}$ de composto $\left(153 \mathrm{e} 135 \mathrm{mg} \mathrm{dm}^{-3}\right.$, respectivamente), conforme pode ser observado na Tabela 1 . Estimou-se que, para cada $100 \mathrm{~kg}$ de composto aplicado por planta, houve aumento de $111 \mathrm{mg} \mathrm{dm}^{-3}$ de $\mathrm{P}$ no solo. E mesmo sendo o $\mathrm{P}$ o macronutriente menos absorvido pela bananeira, sua manutenção no solo é muito importante, pois $50 \%$ do $\mathrm{P}$ absorvido pela planta é exportado pelos frutos (282 $\mathrm{g} \mathrm{t}^{-1}$ de frutos, segundo Hiroce et al., 1977), o que, ao longo dos ciclos, pode gerar deficiência deste elemento, afetando o crescimento vegetativo e também diminuindo o desenvolvimento de raízes (Borges \& Oliveira, 2000).

O potássio e o magnésio no solo foram dois elementos que não foram afetados pelos tratamentos aplicados $(P>0,05)$, apresentando teores médios ao final do experimento de $1,3 \mathrm{mmol}_{\mathrm{c}}$ $\mathrm{dm}^{-3}$ de Ke $20 \mathrm{mmol}_{\mathrm{c}} \mathrm{dm}^{-3}$ de $\mathrm{Mg}$ (Tabela 1). Em função das doses de composto aplicadas, era de se esperar diferenças no teor no solo, principalmente para o potássio, que é o nutriente de maior importância para a bananeira (Borges \& Oliveira, 2000), que corresponde a aproximadamente $41 \%$ do total de nutrientes na planta (que pode variar de 379 a 718,5 g planta $^{-1}$, segundo Borges \& Silva, 1995). Uma 
hipótese para explicar o ocorrido é que a planta tenha absorvido o potássio de forma diferente entre os tratamentos e distribuído na família (mãe, filha e neta). Para o magnésio, pode-se sugerir que o cálcio ou mesmo o potássio tenham deslocado o $\mathrm{Mg}$ do complexo de troca, favorecendo a lixiviação desse nutriente. Apesar disso, através de análise foliar, não foi observado o aparecimento do "azulda-bananeira", que é como é conhecida a deficiência de Mg induzida pela aplicação excessiva de K.

Como foi observado por Damatto Junior (2005), cerca de $81 \%$ do potássio contido no composto orgânico (produzido com serragem de madeira e esterco bovino) foi liberado logo após sua aplicação no solo (22 dias), o que reforça a questão do deslocamento de magnésio e também sugere que parte do próprio potássio tenha também sido lixiviado.

Para o cálcio, houve aumento linear nos teores do solo em função da adição do composto (Tabela 1), sendo que os menores teores foram encontrados na testemunha e no tratamento com a menor dose de adubação (43 $\mathrm{kg}$ planta $^{-1}$ de composto), enquanto nas maiores doses de adubação (129 e $172 \mathrm{~kg}_{\text {planta }}{ }^{-1}$ de composto) encontraram-se os maiores teores, mostrando que o composto produzido foi um bom fornecedor de cálcio ao solo, uma vez que o composto apresentava três vezes mais cálcio que potássio.

A soma de bases (SB), bem como a capacidade de troca catiônica (CTC) foram influenciadas pelos tratamentos, apresentando aumento linear em função das doses de composto, podendo ser observado na Tabela 1 que a testemunha apresentou os menores valores para estes parâmetros, e com os tratamentos, conforme se elevou as quantidades de composto aplicado, esses valores aumentaram, atingindo os valores máximos no tratamento onde foi aplicada a maior quantidade de composto orgânico (172 kg planta $^{-1}$ de composto).

A saturação por bases (V) foi bastante influenciada pelos tratamentos, apresentando seu menor índice na testemunha (69\%), onde não se aplicou composto. Houve aumento linear na saturação por bases do solo, atingindo na maior dose $84 \%$. Para a bananeira, no Estado de São Paulo, a saturação por bases deve ser elevada a 60\% (Raij et al., 1997), já no Espírito Santo, deve ser elevada a 70\%, o que foi conseguido já nas menores doses aplicadas. $\mathrm{O}$ alto valor da saturação por bases atingido neste experimento ocorreu possivelmente pela realização de calagem na instalação do bananal e também pela matéria orgânica (aplicada em forma de composto) elevar o pH devido à adição de resíduos orgânicos e também por adsorver hidrogênio e alumínio na superfície do material orgânico.

Com relação aos micronutrientes, não foi observada diferença significativa entre os tratamentos, sendo que os teores no solo foram considerados altos (Raij et al., 1997), com exceção do boro, que apresentou teor médio (Tabela 2); contudo, pela análise foliar, verificou-se que o boro apresentou teor médio nas folhas, e os demais micronutrientes encontravam-se dentro da faixa considerada ideal para a cultura.

TABELA 1 - Propriedades químicas de solo cultivado com bananeira 'Prata-anã', adubadas com diferentes doses de composto orgânico, em Botucatu-SP (nov/2002 a maio/2004).

\begin{tabular}{|c|c|c|c|c|c|c|c|c|c|c|}
\hline $\begin{array}{l}\text { Dose de composto } \\
\text { kg planta }^{-1}\end{array}$ & $\underset{\mathrm{CaCl}_{2}}{\mathrm{pH}}$ & $\begin{array}{l}\text { M.O. } \\
\text { g/dm }\end{array}$ & $\begin{array}{c}\text { P-res } \\
\mathbf{m g} / \mathbf{d m}^{3}\end{array}$ & $\mathbf{H}+\mathbf{A l}$ & K & $\underset{\text { mmol }_{\mathbf{c}} / \mathbf{d m}^{3}}{\mathbf{C a}}$ & Mg & SB & CTC & $\begin{array}{l}\text { V\% } \\
(\%)\end{array}$ \\
\hline T1: 0 & $5,4 \mathrm{c}$ & $32 \mathrm{~b}$ & $54 \mathrm{~b}$ & $33 \mathrm{a}$ & $1,2 \mathrm{a}$ & $59 \mathrm{~b}$ & $17 \mathrm{a}$ & $77 \mathrm{~d}$ & $108 \mathrm{~d}$ & $\begin{array}{ll}69 \mathrm{c} \\
\end{array}$ \\
\hline $\mathrm{T} 2: 43$ & $5,6 \mathrm{bc}$ & $33 \mathrm{~b}$ & $85 \mathrm{ab}$ & $28 \mathrm{ab}$ & $1,3 \mathrm{a}$ & $74 \mathrm{~b}$ & $19 \mathrm{a}$ & $93 \mathrm{~cd}$ & $124 \mathrm{~cd}$ & $73 \mathrm{bc}$ \\
\hline T3: 86 & $5,7 \mathrm{abc}$ & $35 \mathrm{ab}$ & $93 \mathrm{ab}$ & $27 \mathrm{ab}$ & $1,3 \mathrm{a}$ & $90 \mathrm{ab}$ & $22 \mathrm{a}$ & $113 \mathrm{bc}$ & $141 \mathrm{bc}$ & $79 \mathrm{ab}$ \\
\hline T4: 129 & $6,0 \quad a$ & $43 \mathrm{a}$ & 153 a & $24 \mathrm{~b}$ & $1,4 \mathrm{a}$ & $114 \mathrm{a}$ & $22 \mathrm{a}$ & $137 \mathrm{ab}$ & $161 \mathrm{ab}$ & 85 a \\
\hline T5: 172 & $5,9 \mathrm{ab}$ & $40 \mathrm{ab}$ & $135 \mathrm{ab}$ & $24 \mathrm{~b}$ & $1,3 \mathrm{a}$ & $119 \mathrm{a}$ & $19 \mathrm{a}$ & $151 \quad \mathrm{a}$ & $178 \mathrm{a}$ & $84 \quad \mathrm{a}$ \\
\hline Médias & 5,7 & 37 & 104 & 27 & 1,3 & 91 & 20 & 114 & 142 & 78 \\
\hline Teste F & $8,64^{* *}$ & $5,65^{* *}$ & $4,29^{*}$ & $5,58^{* *}$ & $0,21^{\mathrm{ns}}$ & $9,61^{* *}$ & $1,63^{\mathrm{ns}}$ & $14,76^{* *}$ & $26,19^{* *}$ & $14,78^{* *}$ \\
\hline $\mathrm{CV}(\%)$ & 3,01 & 12,16 & 41,38 & 13,13 & 18,95 & 20,26 & 18,10 & 15,46 & 8,63 & 5,11 \\
\hline
\end{tabular}

Médias seguidas por letras distintas na coluna diferem pelo teste de Tukey, a $5 \%$ de probabilidade.

TABELA 2 - Teores médios de micronutrientes de solo cultivado com bananeira 'Prata-anã', adubadas com diferentes doses de composto orgânico, em Botucatu-SP (nov/2002 a maio/2004).

\begin{tabular}{lccccc}
\hline $\begin{array}{l}\text { Dose de composto } \\
\text { kg planta }^{-1}\end{array}$ & Boro & Cobre & $\begin{array}{c}\text { Ferro } \\
\text { mg/dm }\end{array}$ & Manganês & Zinco \\
\hline T1: 0 & $0,34 \mathrm{a}$ & $9,4 \mathrm{a}$ & $37 \mathrm{a}$ & $13,6 \mathrm{a}$ & $4,5 \mathrm{a}$ \\
T2: 43 & $0,33 \mathrm{a}$ & $7,4 \mathrm{a}$ & $34 \mathrm{a}$ & $12,0 \mathrm{a}$ & $3,6 \mathrm{a}$ \\
T3: 86 & $0,28 \mathrm{a}$ & $7,7 \mathrm{a}$ & $35 \mathrm{a}$ & $12,0 \mathrm{a}$ & $4,0 \mathrm{a}$ \\
T4: 129 & $0,35 \mathrm{a}$ & $7,8 \mathrm{a}$ & $34 \mathrm{a}$ & $12,3 \mathrm{a}$ & $4,8 \mathrm{a}$ \\
T5: 172 & $0,29 \mathrm{a}$ & $7,1 \mathrm{a}$ & $34 \mathrm{a}$ & $12,7 \mathrm{a}$ & $4,5 \mathrm{a}$ \\
\hline Médias & 0,32 & 7,9 & 35 & 12,5 & 4,3 \\
\hline Teste F & $0,62^{\mathrm{ns}}$ & $2,14^{\mathrm{ns}}$ & $0,15^{\mathrm{ns}}$ & $0,50^{\mathrm{ns}}$ & $1,35^{\text {ns }}$ \\
\hline CV $(\%)$ & 28,73 & 17,58 & 18,76 & 16,53 & 20,66 \\
\hline
\end{tabular}

Médias seguidas por letras iguais na coluna não diferem pelo teste de Tukey, a 5\% de probabilidade. 
Diante das melhorias observadas no solo, como manutenção do $\mathrm{pH}$ dentro de uma faixa adequada, elevação nos teores da matéria orgânica, do fósforo e do cálcio no solo, bem como da soma de bases, CTC e saturação por bases, obteve-se produtividade média de $26,24 \mathrm{t} \mathrm{ha}^{-1}$, que pode ser considerada acima da média nacional (13,56 t ha-1), segundo dados da FAO (2006). Contudo, os parâmetros de produção avaliados não mostraram diferença significativa para os tratamentos, sendo encontrado peso médio de cachos de 16,4 $\mathrm{kg}$, com 123 frutos e 9,1 pencas cada.

Mesmo sendo observadas alterações em algumas características químicas do solo, a adubação orgânica não promoveu diferença nas concentrações de nutrientes nas folhas das bananeiras, contudo seus valores encontravam-se dentro de uma faixa tida como adequada para a cultura. Os teores de nutrientes na massa seca foliar mostraram a seguinte ordem de concentração no florescimento das plantas: $\mathrm{K}>\mathrm{N}>\mathrm{Ca}>\mathrm{Mg}>\mathrm{S}>\mathrm{P}(31>30>9>3,2>2,5>2,0$ $\mathrm{g} \mathrm{kg}^{-1}$, respectivamente).

A adubação orgânica proveniente da compostagem promoveu incrementos no $\mathrm{pH}$, matéria orgânica, fósforo, cálcio, soma de bases, CTC e saturação por bases da camada superficial do solo cultivado com bananeira.

\section{AGRADECIMENTOS}

À FAPESP - Fundação de Amparo à Pesquisa do Estado de São Paulo, pela concessão da bolsa de mestrado, processo $\mathrm{n}^{\circ}$ 02/ $11484-4$.

\section{REFERÊNCIAS}

AGRIANUAL 2001: anuário estatístico da agricultura brasileira. São Paulo: FNP Consultoria \& Comercio, 2000. 492p.

AGRIANUAL 2006: anuário estatístico da agricultura brasileira. São Paulo: FNP Consultoria \& Comercio, 2006. 504p.

BARTZ, H.R. (Cood.). Recomendações de adubação e calagem para os estados de Rio Grande do Sul e Santa Catarina. 3.ed. Passo Fundo: SBCS-núcleo Regional Sul, 1995. 224p.

BORGES, A.L.; OLIVEIRA, A.M.G. Nutrição Calagem e Adubação. In: CORDEIRO, Z.J.M. Banana: produção: aspectos técnicos. Brasília: Embrapa Comunicação para Transferência de Tecnologia, 2000. p.47-59. (Frutas do Brasil; 1).

BORGES, A.L.; SILVA, S.O. Extração de macronutrientes por cultivares de banana. Revista Brasileira de Fruticultura, Cruz das Almas, v.17, n.1, p.57-66, 1995.

CAMPO DALL'ORTO, F.A. et al. Frutas de clima temperado II: Figo, maçã, marmelo, pêra e pêssego em pomar compacto. In: RAIJ, B. van. et al. (Ed.). Recomendações de adubação e calagem para o Estado de São Paulo. 2.ed. Campinas: Fundação Instituto Agronômico, 1996, p.139-140.

CARVALHO, W.A.; ESPÍNDOLA, C.R.; PACCOLA, A.A. Levantamento de solos da Fazenda Lageado - Estação Experimental "Presidente Médici". Boletim Científico Faculdade de Ciências Agronômicas, Botucatu, v.1, p.1-95, 1983.

COMISSÃO DE FERTILIDADE DO SOLO DOESTADODE MINAS GERAIS. Recomendações para o uso de corretivos e fertilizantes em Minas Gerais, 4. aproximação. Lavras, 1989. 176p.

CORDEIRO, Z.J.M. Doenças. In: ALVES, E.J. A cultura da banana: aspectos técnicos, socioeconômicos e agroindustriais. 2.ed. rev. Brasília: Embrapa-SPI/ Cruz das Almas: Embrapa-CNPMF, 1999. p353-408.

CUNHA, A.R.; KLOSOWSKI, E.S.; GALVANI, E.; SCOBEDO, J.F.; MARTINS, D. Classificação climática para o município de Botucatu-SP, segundo Koppen. In: SIMPÓSIO EM ENERGIA NA AGRICULTURA, 1., 1999, Botucatu. Anais... Botucatu: Faculdade de Ciências Agronômicas, Universidade Estadual Paulista, 1999. p.487-491.
DAMATTO JUNIOR, E.R. Efeitos da adubação com composto orgânico na fertilidade do solo, desenvolvimento, produção e qualidade de frutos de bananeira 'Prata-anã' (Musa AAB). 2005. 70f. Dissertação (Mestrado em Agronomia / Energia na Agricultura) - Faculdade de Ciências Agronômicas, Universidade Estadual Paulista, Botucatu, 2005.

EMBRAPA. Centro Nacional de Pesquisa de Solos. Sistema Brasileiro de Classificação de Solos. Rio de Janeiro: EMBRAPA/ SOLOS, 1999.412p.

FAO-FOOD AND AGRICULTURE ORGANIZATION. Disponível em: 〈http://faostat.fao. org/faostat>. Acesso em: 16 jul. 2006.

GALLO, J.R.; BATAGLIA, O.C.; FURLANI, P.R.; HIROCE, R.; FURLANI, A.M.C.; RAMOS, M.T.B.; MOREIRA, R.S. Composição química inorgânica da bananeira (Musa acuminata), cultivar nanicão. Ciência e Cultura, São Paulo, v.24, n.1, p.70-79, 1972.

HEWITT, C.W. Leaf analysis as a guide to the nutrition of bananas. Empire Journal of Experimental Agriculture, London, v.23, n.89, p.11-16, 1955.

HIROCE, R.; CARVALHO, A.M. de; BATAGLIA, O.C.; FURLANI, P.R.; FURLANI, A.M.C.; SANTOS, R.R. dos; GALLO, J.R. Composição mineral de frutos tropicais na colheita. Bragantia, Campinas, v.36, n.14, p.155-164, 1977.

HOYT, P.B.; TURNER, R.C. Effect of organic materials added to very acid soils on $\mathrm{pH}$, aluminum, exchangeable $\mathrm{NH}_{4}$ and crop yelds. Soil Science, Baltimore, v.119, p.227-37, 1975.

HUNTER, D.J.; YAPA, L.G.G.; HUE, N.V.; EAQUB, M. Comparative effects of green manure and lime on the growth of sweet corn and chemical properties of an acid oxisol in Western Samoa. Communications in Soil Science and Plant Analysis, Philadelphia, v.26, p.375-88, 1995.

KIEHL, E.J. Fertilizantes orgânicos. Piracicaba: Agronômica Ceres. 1985. 492p.

LARCHER, W. Ecofisiologia Vegetal. São Carlos: Rima Artes e Textos, 2000. 531p.

MARTIN-PRÉVEL, P. Os elementos minerais da bananeira e dos seus frutos. Fertilité, Paris, v.22, p.3-14, 1964.

MOREIRA, R.S. Banana: teoria e prática de cultivo. Campinas: Fundação Cargill, 1987. 335p.

NEVES, R.L.L.; FERREIRA, F.F.H.; MACIEL, R.E.P.; FROTA, J.N.E. Extração de nutrientes em banana (Musa sp.) cv. Pacovan. Ciência Agronômica, Fortaleza, v.22, n.1/2, p.115-120, 1991.

RAIJ, B.; Quaggio, J.A. Métodos de análises de solo para fins de fertilidade. Campinas; Instituto Agronômico, 1983. p.1-31. (Boletim Técnico, 81).

RAIJ, B. van; CANTARELLA, H.; QUAGGIO, J.A.; FURLANI, A.M.C. (Ed.). Recomendações de adubação e calagem para o Estado de São Paulo. 2.ed. rev. e atual. Campinas: Instituto Agronômico/Fundação IAC, 1997. 285p. (Boletim Técnico, 100).

TWYFORD, I.T.; WALMSLEY, D. The mineral composition of the robusta banana plant. III. Uptake and distribution of mineral constituents. Plant and Soil, Dordrecht, v.41, n.3, p.471-491, 1974.

WONG, M.T.F.; AKEAMPONG, E.; NORTCLIFF, S.; RAO, M.R.; SWIFE, R.S. Initial responses of maize and beans to decreased concentrations of monomeric inorganic aluminium with application of manure or tree prunings to an oxisol in Burundi. Plant and Soil, Dordrecht, v.171, p.275-82, 1995. 